\title{
Correction to: Ruptured posterior circulation aneurysms: epidemiology, patterns of care, and outcomes from the Swiss SOS national registry
}

\author{
Rodolfo Maduri ${ }^{1}$ D - Daniele Starnoni ${ }^{1}$ - Alda Rocca ${ }^{1}$ - David Bervini ${ }^{2}$ - Daniel Walter Zumofen ${ }^{3,4}$. \\ Martin Nikolaus Stienen ${ }^{5}$. Bawarjan Schatlo ${ }^{6}$. Christian Fung ${ }^{7} \cdot$ Thomas Robert $^{8}$ - Martin A. Seule ${ }^{9}$. \\ Jan-Karl Burkhardt ${ }^{5,10}$. Nicolai Maldaner ${ }^{5}$. Michel Rothlisberger ${ }^{3} \cdot$ Kristine A. Blackham $^{4}$. Serge Marbacher ${ }^{11}$. \\ Donato D'Alonzo ${ }^{11}$. Luca Remonda ${ }^{12}$. Paolo Machi ${ }^{13}$ • Jan Gralla ${ }^{14}$. Philippe Bijlenga ${ }^{15}$. Guillaume Saliou ${ }^{16}$. \\ Pierluigi Ballabeni ${ }^{17,18} \cdot$ Marc Levivier $^{1,18} \cdot$ Mahmoud Messerer ${ }^{1,18} \cdot$ Roy Thomas Daniel $^{1,18} \cdot$ Swiss SOS Group
}

Published online: 23 February 2019

(C) Springer-Verlag GmbH Austria, part of Springer Nature 2019

\section{Correction to: Acta Neurochirurgica https://doi.org/10.1007/s00701-019-03812-9}

The name of Roy Thomas Daniel was incorrectly captured in the original manuscript.

The correct authorname should be:

$<$ GivenName $>$ Roy $<$ /GivenName $>$

$<$ GivenName $>$ Thomas $<$ GivenName $>$

$<$ FamilyName>Daniel</FamilyName $>$

The online version of the original article can be found at https://doi.org/10. 1007/s00701-019-03812-9

Rodolfo Maduri

Rodolfo.maduri@gmail.com

1 Department of Clinical Neurosciences, Service of Neurosurgery, Lausanne University Hospital (CHUV), 46 rue du Bugnon, 1011 Lausanne, Switzerland

2 Department of Neurosurgery, Inselspital, University of Bern, Bern, Switzerland

3 Department of Neurosurgery, Basel University Hospital, Basel, Switzerland

4 Diagnostic and Interventional Neuroradiology Section, Department of Radiology, Basel University Hospital, Basel, Switzerland

5 Department of Neurosurgery, University Hospital of Zurich \& Clinical Neurosciences Center, University of Zurich, Zurich, Switzerland

6 Department of Neurosurgery, University Hospital Göttingen, Göttingen, Germany

7 Department of Neurosurgery, Medical Center, University of Freiburg, Freiburg, Germany

8 Department of Neurosurgery, Ospedale Civico di Lugano, Lugano, Switzerland
The original article has been corrected.

Publisher's note Springer Nature remains neutral with regard to jurisdictional claims in published maps and institutional affiliations.

9 Department of Neurosurgery, Kantonsspital St. Gallen, St. Gallen, Switzerland

10 Department of Neurosurgery, Baylor Medical Center \& College of Medicine, Houston, TX, USA

11 Department of Neurosurgery, Kantonsspital Aarau, Aarau, Switzerland

12 Department of Radiology, Division of Diagnostic and Interventional Neuroradiology, Kantonsspital Aarau, Aarau, Switzerland

13 Department of Radiology, Division of Diagnostic and Interventional Neuroradiology, Geneva University Hospital (HUG), Geneva, Switzerland

14 Department of Radiology, Division of Diagnostic and Interventional Neuroradiology, Inselspital, University of Bern, Bern, Switzerland

15 Department of Neurosurgery, Hopitaux Universitaires Genève, Geneva, Switzerland

16 Department of Radiology, Division of Diagnostic and Interventional Neuroradiology, Lausanne University Hospital (CHUV), Lausanne, Switzerland

17 Lausanne Institute for Clinical Epidemiology and Biostatistics, University Hospital Lausanne (CHUV), Lausanne, Switzerland

18 University of Lausanne (UniL), Lausanne, Switzerland 